BMJ

Open

Gastroenterology

\title{
Use of ethylene-oxide gas sterilisation to terminate multidrug-resistant bacterial outbreaks linked to duodenoscopes
}

\author{
Lawrence F Muscarella
}

To cite: Muscarella LF. Use of ethylene-oxide gas sterilisation to terminate multidrugresistant bacterial outbreaks linked to duodenoscopes. BMJ Open Gastro 2019;6:e000282. doi:10.1136/ bmjgast-2019-000282

Received 4 February 2019 Revised 9 May 2019 Accepted 10 May 2019
Check for updates

(C) Author(s) (or their employer(s)) 2019. Re-use permitted under CC BY-NC. No commercial re-use. See rights and permissions. Published by BMJ.

LFM Healthcare Solutions, LLC, Montgomeryville, Pennsylvania, USA

Correspondence to Lawrence F Muscarella; Larry@LFM-HCS.com

\section{ABSTRACT}

Background Cleaning and high-level disinfection have been the standard in the USA and other countries for reprocessing flexible endoscopes, including duodenoscopes and other types of gastrointestinal endoscopes. For decades, this practice has been a cornerstone for infection prevention in the endoscopic setting. However, amid recent reports associating the use of duodenoscopes with infections and outbreaks of carbapenem-resistant Enterobacteriaceae (CRE) and related multidrug-resistant organisms (MDROs), reasonable questions about the adequacy of current practices for reprocessing duodenoscopes have emerged. Objectives To review and evaluate the adequacy of current reprocessing practices for preventing duodenoscopes from transmitting CRE and related MDROs. Methods The MEDLINE/PubMed database was searched to identify published cases associating confirmed (or suspected) infections of CRE or a related MDRO with exposure to a duodenoscope since 2012, when duodenoscopes became a recognised risk factor for the transmission of CRE. The Internet was also searched to identify news articles and other reports documenting eligible cases occurring during this same timeframe but not identified during the MEDLINE database's search. The Food and Drug Administration's (FDA) medical device database was queried to identify regulatory reports describing these same types of cases, also recorded since 2012. The clinical and reprocessing details of each eligible case were reviewed to identify (when possible): (a) the reprocessing method (eg, high-level disinfection) performed at the time of the infections, (b) whether the facility's compliance with the manufacturer's reprocessing instructions was confirmed, and (c) the measure(s) or corrective action(s) the facility implemented to prevent additional multidrug-resistant infections.

Results Seventeen cases in the USA and six in other countries (primarily Europe) associating infections (and colonizations) of CRE or a related MDRO with exposure to a duodenoscope were reviewed. Fourteen of these 23 outbreaks were caused by CRE, and six by a related MDRO. Two of these six latter cases identified Klebsiella pneumoniae carrying the $m c r-1$ gene as the pathogen. For 12 of these 23 cases, it was reported or implied that the duodenoscope was being high-level disinfected at the time of the infections, consistent with published guidelines. For the remaining 11 cases, the associated report(s) did not clearly identify how the duodenoscope was being reprocessed at the time of the infections (although it may be reasonably concluded that at least some, if not all, of these 11 cases involved high-level disinfection).

Further, eight of the 23 cases reported the duodenoscope was being reprocessed in accordance with the manufacturer's instructions for use (and professional guidelines) at the time of the infections. Seven of the cases discussed the design of the duodenoscope (eg, the forceps elevator mechanism) in the context of reprocessing and the infections. Three of the cases identified one or more reprocessing lapses, including inadequate cleaning, delayed reprocessing and improper drying and/or storage of the duodenoscope. Most of these 23 cases were associated with exposure to a duodenoscope model featuring a sealed elevator-wire channel. Six of the cases reported adopting (or in one case supplementing high-level disinfection with) ethylene oxide (E0) gas sterilisation of the duodenoscope, with at least three reporting this measure terminated the outbreak. Other measures adopted to prevent additional infections included removing the implicated duodenoscope from use, re-training staff about proper cleaning, microbiological culturing of the duodenoscope and returning the duodenoscope to the manufacturer for evaluation, maintenance and/or repair.

Conclusions This study's findings suggest current reprocessing practices may not always be sufficiently effective to prevent a duodenoscope from transmitting CRE and related MDROs, at least in some circumstances including an outbreak setting. Factors this review identified that may contribute to the device remaining contaminated after reprocessing include the device's design; breaches of recommended reprocessing guidelines (eg, inadequate manual cleaning, delayed reprocessing or improper device storage); damage to the device; lacking servicing, maintenance or repair; and/or the presence of biofilms. Measures that can mitigate the impact of these and other reprocessing challenges and reduce, if not eliminate, the risk of transmission of CRE or a related MDRO by a duodenoscope include the use of $E 0$ gas sterilization (or another comparably effective process or method). In 2015, the FDA suggested healthcare facilities consider performing at least one of four supplemental measures, which include EO gas sterilisation, to improve the effectiveness of duodenoscope reprocessing. Whether the FDA and Centers for Disease Control and Prevention might reclassify duodenoscopes as critical devices requiring sterilisation is currently unresolved. 


\section{BACKGROUND}

Cleaning and high-level disinfection have been the standard in the USA and other countries for reprocessing duodenoscopes and other gastrointestinal (GI) endoscopes. ${ }^{1-3}$ This practice, known as reprocessing, is derived from a three-tiered scheme, developed almost 50 years ago by Spaulding, ${ }^{4}$ that classifies devices into one of three categories depending on the potential infection risk. The category into which the device is classified dictates the necessary level of disinfection, or sterilisation. Invasive surgical instruments that enter sterile tissues or the vascular system, such as cardiac catheters, pose a high risk of infection and therefore are classified as critical devices requiring sterilisation. ${ }^{1}$ In contrast, noncritical devices such as stethoscopes pose a low infection risk, as they contact only intact skin (but not mucous membranes). ${ }^{1}$ The Food and Drug Administration (FDA) recommends thorough cleaning followed by low-level or intermediatelevel disinfection for these low-risk devices (depending on the nature and extent of contamination). ${ }^{5}$ Low-level disinfection destroys most vegetative bacteria, some fungi and some viruses, but neither Mycobacterium tuberculosis nor bacterial endospores. Intermediate-level disinfection is tuberculocidal, but not sporicidal. ${ }^{1}$

Duodenoscopes and most other flexible endoscopes are classified as semicritical devices. ${ }^{1}$ These devices generally contact non-intact skin or intact mucous membranes requiring high-level disinfection or sterilisation. Primarily because duodenoscopes are damaged by pressurised steam, high-level disinfection achieved using a liquid chemical has been the practiced standard for decades. ${ }^{1-12}$ Nevertheless, sterilisation would be ideal, understanding not only that sterilisation provides a greater margin of safety, but also that duodenoscopes (and other heatsensitive semicritical devices) may occasionally contact blood or a sterile accessory (eg, during a biopsy). ${ }^{1451314}$ The FDA recommends sterilisation for semicritical devices, but accepts high-level disinfection "[i]f the device design does not permit sterilization (eg, device materials cannot withstand sterilization)." ${ }^{5}$ While high-level disinfection destroys virtually all pathogenic microorganisms encountered in the endoscopic setting, ${ }^{1}$ the more robust process of sterilisation renders the device free of all viable microorganisms. ${ }^{14}$

In the wake of several recently published reports associating infections of carbapenem-resistant Enterobacteriaceae (CRE) or a related multidrug-resistant organism (MDRO) with exposure to a duodenoscope, reasonable questions may be asked about the effectiveness of current reprocessing practices. ${ }^{7-20}$ The potential significance of these questions is spotlighted by the FDA's recent safety communications discussing duodenoscopes, and the agency's statement that these devices feature a forceps elevator mechanism whose moving parts contain "microscopic crevices that may not be reached with a brush" and may remain contaminated after cleaning and disinfection. ${ }^{591314}$ The FDA has further advised that cleaning a duodenoscope prior to high-level disinfection may not entirely eliminate the risk of transmitting infection. ${ }^{13} 14$

The implementation of validated measures to prevent duodenoscopes from transmitting multidrug-resistant bacteria is crucial to public health because these devices are used more than 500000 times a year in the USA to perform endoscopic retrograde cholangiopancreatography, or ERCP - a medical procedure used to examine and treat disorders of the bile and pancreatic ducts. ${ }^{813}$ Efforts to improve safety are aided by the study of these outbreaks to evaluate current reprocessing practices, identify common factors that can contribute to infection, and publish effective corrective actions that improve safety. (Note: "Linking" or "associating" a duodenoscope or another type of reusable device with an infection or an outbreak does not confirm the device transmitted or otherwise caused the infection, as one or more other factors could be, in part or solely, responsible. More data would be required to conclude more definitively that a device caused an infection.)

\section{OBJECTIVES}

To review and evaluate the adequacy of current reprocessing practices for preventing duodenoscopes from transmitting CRE and related MDROs during ERCP.

\section{METHODS}

As similarly described in another study, ${ }^{21}$ the MEDLINE/ PubMed database was searched using the MeSH (medical subject headings) terms duodenoscope, disease outbreak and bacteria to identify published cases associating confirmed (or suspected) infections of CRE or a related MDRO with exposure to a duodenoscope since 2012, when duodenoscopes became a recognised risk factor for the transmission of CRE. ${ }^{7-12}$ The Internet was searched using these same keywords (via Google's search engine) to identify news articles and other reports documenting eligible cases occurring during this same timeframe but not identified during the MEDLINE database's search. The FDA's Manufacturer and User Facility Device Experience Database (MAUDE) was queried using the product code "FDT" (which the FDA and manufacturers generally use to refer to duodenoscopes) to identify regulatory reports describing these same types of cases also recorded since 2012. ${ }^{5}$ (Note: The findings and conclusions of the regulatory reports in the MAUDE database generally have not been peer-reviewed and may be incomplete and/ or in part inaccurate. Nevertheless, these reports can yield important safety data that can be used to monitor a device's performance and to detect potential risks.) ${ }^{14}$

Guidelines published by the Preferred Reporting Items for Systematic Reviews and Meta-Analyses were used to screen these search results, exclude duplicate cases and identify only reports relevant to this study's objective. In general, this review excluded reports linking a duodenoscope to an infection caused by an organism other than CRE or a related MDRO, or a report describing bacterial 
contamination of a duodenoscope but without sufficient evidence of an infection. The clinical and reprocessing details of each eligible case were reviewed to identify (when possible): (a) the reprocessing method (eg, high-level disinfection) performed at the time of the infections, (b) whether the facility's compliance with the manufacturer's reprocessing instructions was confirmed, and (c) the specific measure(s) the facility implemented to prevent additional multidrug-resistant infections. Some other details were also investigated, such as whether the duodenoscope featured an open or sealed elevator-wire channel, and whether the implicated duodenoscope was tested (ie, sampled and cultured) for bacterial contamination. Due to inherent limitations with its search methods and other considerations, this systematic review did not include every eligible case, but efforts were made to avoid a selection bias that might have influenced this study's findings and conclusions.

CRE are Enterobacteriaceae that are not susceptible to carbapenem antibiotics; examples include carbapenemresistant strains of Klebsiella pneumoniae, Escherichia coli and Enterobacter cloacae. As similarly defined in other studies, ${ }^{8} 21$ this systematic review defined a MDRO "related" to CRE as a gram-negative, multidrug-resistant bacterium that: $(i)$ demonstrates resistance to carbapenem antibiotics although is not of the Enterobacteriaceae family-for example, Verona Integron-Mediated Metallo- $\beta$-lactamase (VIM)-2-producing Pseudomonas aeruginosa; (ii) is of the Enterobacteriaceae family and resistant to most $\beta$-lactam antibiotics, but remains generally susceptible to carbapenems-for example, extended-spectrum $\beta$-lactamases (ESBL)-producing K. pneumoniae; or (iii) demonstrates resistance to the colistin antibiotic-for example, mor-1-positive K. pneumoniae. Even more so than carbapenems, colistin is a "last-resort" antibiotic used by clinicians to treat serious gram-negative infections caused by multidrug-resistant bacteria (eg, when carbapenems are unsuccessful). CRE and their related MDROs may be informally referred to as "superbugs." 78

\section{RESULTS}

Seventeen cases in the USA and six in other countries (primarily Europe) associating infections (and colonizations) of CRE or a related MDRO with exposure to a duodenoscope were reviewed, and are listed in tables 1 and 2 , respectively. (These tables use a format similar to that provided in other reports. ${ }^{8}{ }^{21}{ }^{22}$ ) Fourteen of these 23 outbreaks were caused by CRE, and six by a related MDRO. Two of these six latter cases identified K. pneumoniae carrying the mcr-1 gene as the pathogen; whether these two cases describe one incident could not be determined. (One of these 23 cases was possibly due to CRE, one likely to related MDROs, and one case either CRE or a MDRO, but it was unclear which one.) For 12 of these 23 cases, it was reported or implied that the duodenoscope was being high-level disinfected at the time of the infections, consistent with published guidelines. ${ }^{1-4}$ For the remaining 11 cases, the associated report(s) did not clearly identify how the duodenoscope was being reprocessed at the time of the infections (although it may be reasonably concluded that at least some, if not all, of these 11 cases involved high-level disinfection).

Further, eight of the 23 cases reported that the infections occurred despite no identifiable breach in the reprocessing procedure (ie, the duodenoscope was reportedly being reprocessed in accordance with manufacturer's instructions for use and professional guidelines). Seven of the cases discussed the design of the duodenoscope (eg, the forceps elevator mechanism) in the context of reprocessing and the infections. Three of the cases reported one or more reprocessing lapses, including inadequate cleaning, delayed reprocessing and improper drying and/or storage of the duodenoscope. Most of these 23 cases were associated with exposure to a duodenoscope (not exclusive to one model or manufacturer) featuring a sealed elevator-wire channel. Six of the cases reported adopting (or in one case supplementing high-level disinfection with) ethylene oxide (EO) gas sterilisation of the duodenoscope, with at least three reporting this measure terminated the outbreak. ${ }^{7-10} 1617202324$ (Whether the use of EO gas sterilization terminated each of the other outbreaks, too, is unclear.) Other measures and mitigations that were adopted to prevent additional infections included removing the implicated duodenoscope from use, re-training staff about proper cleaning, microbiological culturing, reprocessing the duodenoscope twice, and/or returning the duodenoscope to the manufacturer for evaluation, maintenance and/or repair. ${ }^{711} 12$ 25-29

First outbreak of CRE in the USA linked to a duodenoscope: Some additional details of a number of these 23 cases are provided to supplement the data in table 1 and table 2. For instance, Alrabaa et al of Florida reported that seven patients between June 2008 and January 2009 were positive for carbapenemase-producing K. pneumoniae (CRE) following ERCP. ${ }^{29}$ (This case occurred prior to 2012 but whose investigation was not published until 2013, justifying its inclusion in this review.) These investigators associated transmission with "inadequate cleaning of the complex terminal part of the ERCP scope that contains the scope elevator," adding that "[b]iodebris remained under the elevator piece of the implicated scope after it was presumably cleaned." Alrabaa et al also reported that the duodenoscope was "particularly hard to clean," and that the forceps elevator mechanism "needs additional manual cleaning using a brush prior to standard scope processing." No new infections were identified once several measures were implemented, including the education of staff about the proper handling and cleaning of the duodenoscope. This case (beginning in 2008) may be the first to associate a duodenoscope with transmissions of CRE. (The report does not identify the implicated duodenoscope's type or model.)

First outbreak of carbapenem-resistant bacteria linked to a sealed duodenoscope: Another of the case's listed in table 1, Verfaillie et al reported that 22 patients at a hospital in the 


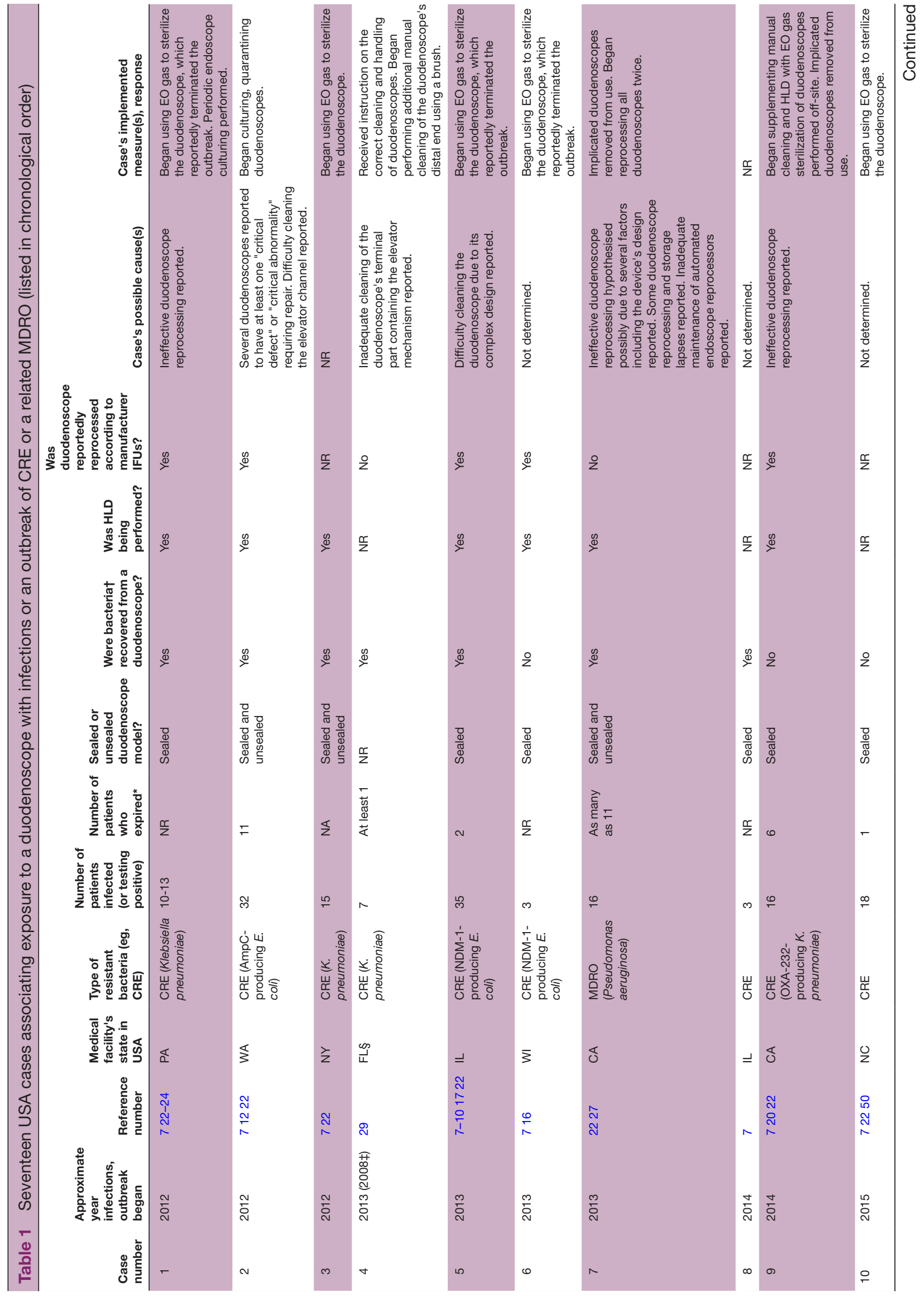




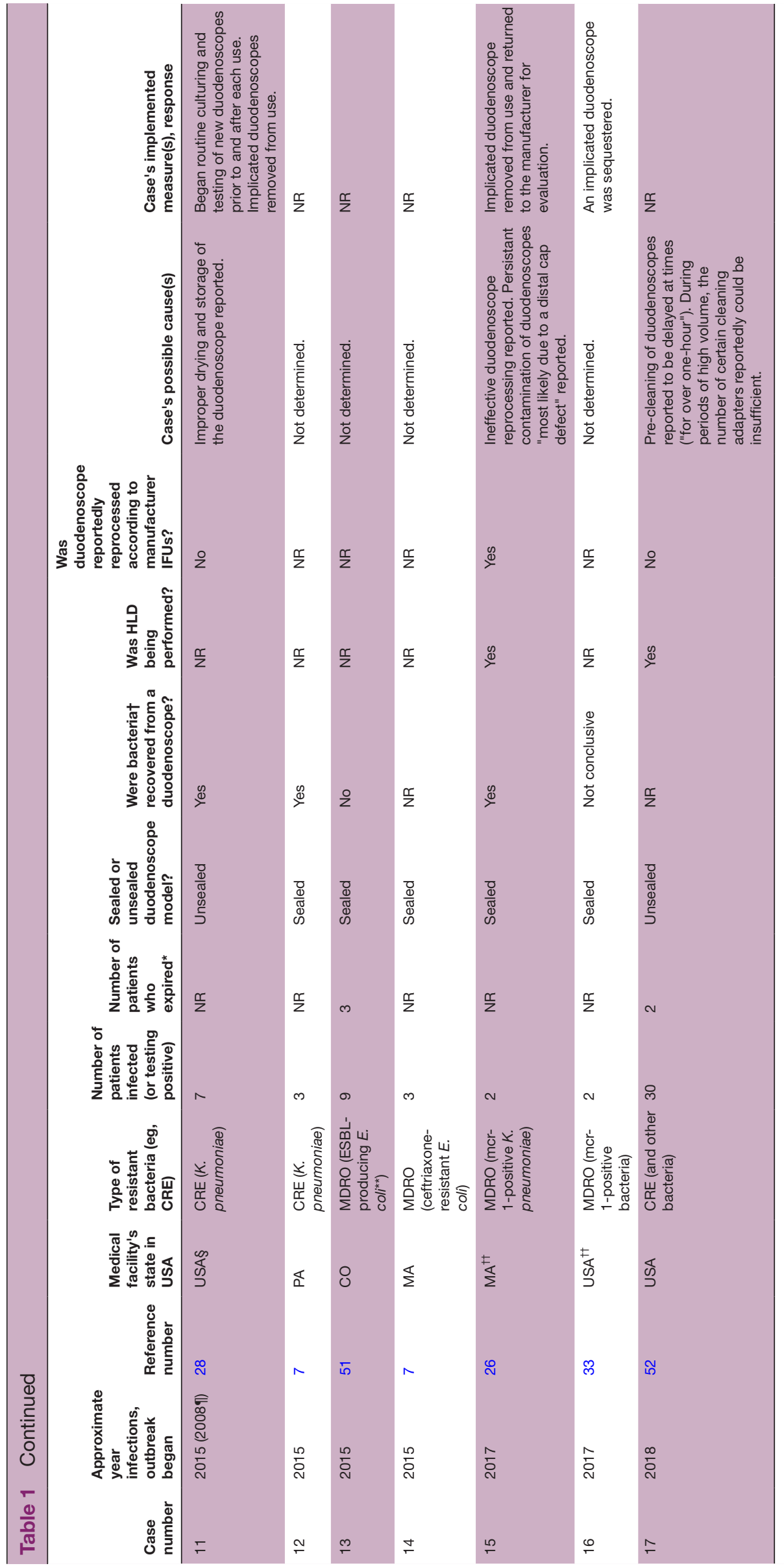




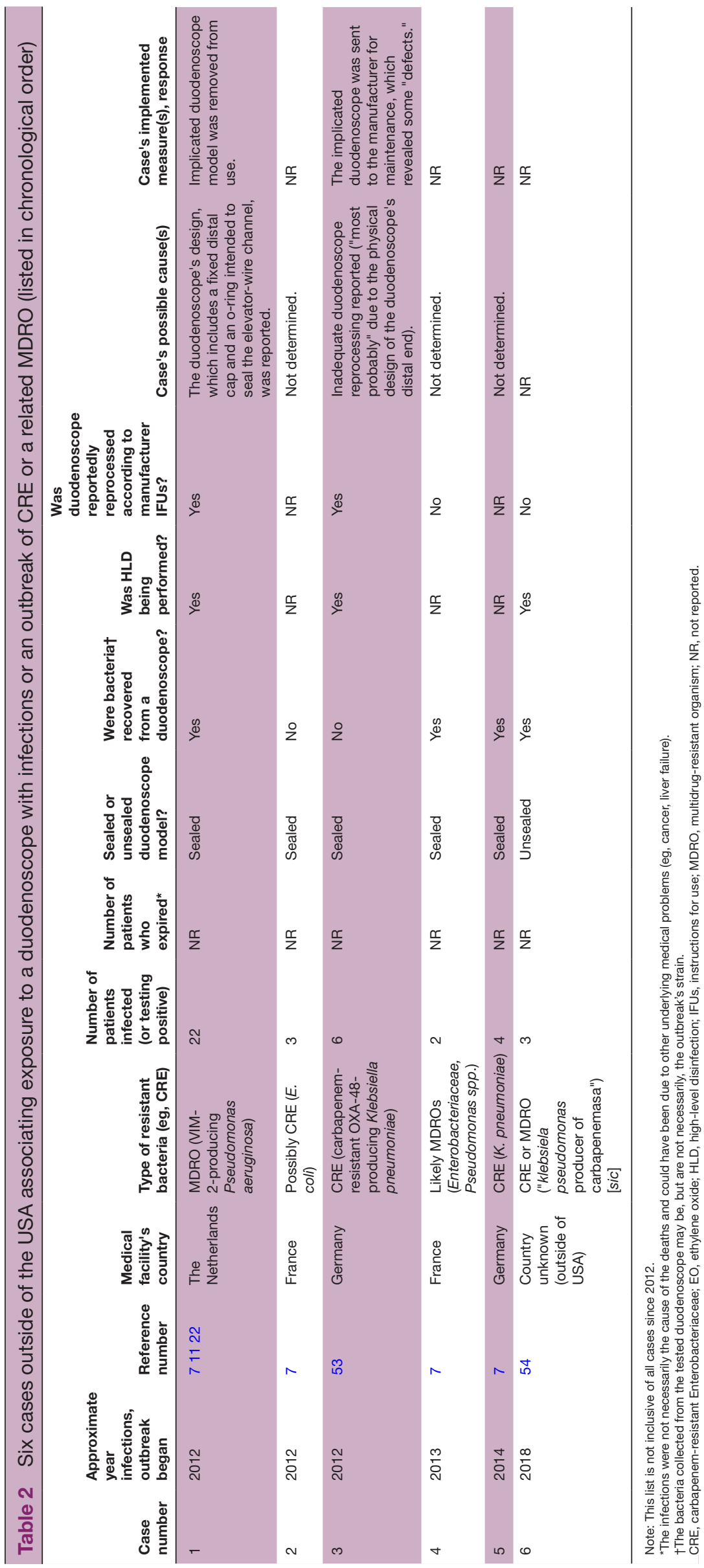


Netherlands tested positive for VIM-2-producing $P$. aeruginosa following ERCP performed between January and April 2012 using a newer duodenoscope model featuring a sealed (or, enclosed) elevator-wire channel. ${ }^{911}$ (This organism is a gram-negative MDRO resistant to carbapenems). Cultures obtained from the recess under the forceps elevator of a high-level disinfected duodenoscope were reported to be clonally related to the outbreak's strain (ie, patient isolates). Verfaillie et al also reported identifying no known breaches in adherence to the manufacturer's duodenoscope reprocessing procedures. ${ }^{11}$ This report may document the first case, either in Europe or the USA, linking use of this newer duodenoscope model to infections of carbapenem-resistant bacteria.

These investigators further reported that the duodenoscope's distal end "hampered cleaning and disinfection," while also raising questions about the construction of this duodenoscope model's elevator-wire channel. ${ }^{11} 22$ The hospital terminated this outbreak by removing this duodenoscope model from clinical use. This outbreak is a salient case because almost all instances of disease transmission associated with a contaminated GI endoscope reported in previous years was attributed to an identifiable lapse in the reprocessing of the endoscope or an accessory (or, either a faulty infection prevention practice such as improper administration of a medication, or the use of defective equipment). 12691029

First publicised outbreak of CRE in the USA linked to a sealed duodenoscope: Almost 2 years after this Netherlands outbreak, the CDC issued a brief report in 2014 linking ERCP to transmissions of New Delhi metallo- $\beta$-lactamase (NDM)-producing E. coli (CRE) at a hospital in Illinois. ${ }^{10}$ Echoing the previous findings of others, ${ }^{29}{ }^{30}$ the CDC reported that the duodenoscope's design "might pose a particular challenge for cleaning and disinfection." Listed in table 1, this case documents the first outbreak of CRE in the USA that federal health officials publicly associated with the exposure to a duodenoscope model featuring an enclosed elevator-wire channel, and who further advised that healthcare facilities "with CRE outbreaks should consider the possibility of ERCPrelated transmission." ${ }^{10}$ No additional infections were identified once the hospital began sterilizing its duodenoscopes with EO gas. ${ }^{7910}$

Epstein et al published more details about this Illinois hospital's outbreak 9 months after issuance of the CDC's brief report, noting that 35 patients who underwent ERCP tested positive in 2013 for the CRE. ${ }^{9}$ Like the CDC's report, ${ }^{10}$ Epstein et al reported no identifiable breaches in the hospital's cleaning or high-level disinfection protocols; however, these investigators reported recovering from the reprocessed duodenoscope CRE closely related to the outbreak's strain. ${ }^{9}$ Positing that sterilisation might have contributed to controlling this outbreak, these investigators acknowledged, however, that "the limited experience from this investigation does not provide sufficient evidence to recommend that all facilities switch to sterilisation." ${ }^{9}$ Four months later in February 2015, the
FDA issued a safety communication advising (consistent with the CDC's findings ${ }^{910}$ ) that recently published data had associated multidrug-resistant bacterial infections in patients following ERCP with duodenoscopes reportedly reprocessed according to the manufacturer's instructions for use. ${ }^{13}$

Three other outbreaks of CRE in the USA: Another of table 1's cases, Humphries et al reported that beginning in December 2014 nine patients in California were found to be infected (and seven asymptomatic carriers) with CRE (ie, carbapenem-resistant $K$. pneumoniae containing the bla $_{\text {OXA-232 }}$ gene) following ERCP. ${ }^{2022} 31$ An investigation that included reviewing the use, reprocessing and storage of duodenoscopes did not identify deviations from manufacturer-provided guidelines. Although cultures obtained from the duodenoscopes yielded negative results, Humphries et al reported that this investigation implicated two specific duodenoscopes featuring a sealed elevator-wire channel. This outbreak of CRE was terminated once the hospital, in addition to permanently removing the implicated duodenoscopes from service, shipped all duodenoscopes off-site to be sterilised using EO gas (after manual cleaning and high-level disinfection)..$^{20} 31$

Smith et alreported that three patients became infected with NDM-1-producing E. colifollowing ERCP performed between May 2013 and November 2013 at a Wisconsin hospital (table 1). ${ }^{16}$ (This is the same type of CRE that was responsible for the aforementioned Illinois outbreak in 2013. ${ }^{9}{ }^{10}$ ) As has been similarly reported about other outbreaks, Smith et al reported the infections occurred despite the hospital having apparently reprocessed the duodenoscope correctly, and that no additional CRE infections were identified once the hospital began sterilizing the duodenoscope using EO gas (table 1). Although they did not recover the outbreak's CRE from the hospital's duodenoscopes, these investigators linked one specific duodenoscope to the outbreak, concluding that "the epidemiologic evidence was strong enough to implicate the duodenoscope as the mode of transmission." ${ }^{16}$ (Note: The failure to recover an outbreak's strain of CRE, or a related MDRO, from a duodenoscope is not entirely uncommon, ${ }^{20} 3032$ and can be due to several factors including the sampling technique's limitations and/or the bacteria's "inaccessibility deep within the elevator channel." ${ }^{22}$ ) Smith et al did not identify the specific duodenoscope model linked to this outbreak, but an independent publication issued in 2016 provided data reporting that the model featured a sealed elevatorwire channel. ${ }^{76}$

McCool et al associated an outbreak of carbapenemresistant $K$. pneumoniae (CRE) at a hospital in Pennsylvania in 2012 with the use of duodenoscopes featuring a sealed elevator channel (table 1). ${ }^{724}$ An investigation reported that these duodenoscopes were being reprocessed consistent with manufacturer instructions, ${ }^{7}$ causing McCool $e t$ al to ask whether is is time to switch to $\mathrm{EO}$ gas sterilisation for reprocessing GI endoscopes featuring an elevator 
channel. ${ }^{24}$ This Pennsylvania outbreak was reportedly terminated once the hospital began sterilising its duodenoscopes using EO gas.

Colistin-resistant bacteria carrying the mor-1 gene: A duodenoscope manufacturer submitted five similar regulatory reports to the FDA in August 2017 (each reasonably and presumably referring to the same case) describing the "probable transmission" of a uniquely resistant type of bacteria that is potentially even more concerning than CRE (table 1). The reports discuss two patients positive for "ctx-r Klebsiella pneumonia" [sic] who both underwent ERCP using a duodenoscope that featured a sealed elevator-wire channel. ${ }^{33}$ According to these reports, the CDC tested the bacteria and determined that, for both patients, the strain carried the mcr-1 gene, which confers the bacteria's resistance to colistin. (Colistin-resistant bacterial infections can be untreatable. $)^{26}$ These regulatory reports further state that the CDC found that the isolates from the two patients and the duodenoscope "appear related." These reports do not identify the measures the medical facility adopted to mitigate the risk of additional infections. This case may be the first to describe a duodenoscope in the context of infections of mor-1-positive K. pneumoniae.

\section{DISCUSSION}

Although sterilisation is more robust, high-level disinfection has been the standard in the USA and other countries for reprocessing flexible endoscopes, including duodenoscopes and other GI endoscopes. ${ }^{1-4}$ For decades, this practice has provided a cornerstone for infection prevention in the endoscopic setting. ${ }^{1-3}$ However, amid the outbreaks of CRE or a related MDRO listed in tables 1 and 2 (among other cases), questions have emerged about the effectiveness of current practices for reprocessing duodenoscopes. ${ }^{7-20}$ Some of these cases reported that implementing EO gas sterilisation of the duodenoscope prevented additional infections. ${ }^{79} 101617202224$ These findings suggest, first, that current reprocessing practices may not always be sufficiently effective to prevent a duodenoscope from transmitting CRE and related MDROs, at least in some circumstances including an outbreak setting. Second, these data also suggest that EO gas sterilization can be an effective option for improving the safety of ERCP. ${ }^{22}$ Factors that may contribute to the device remaining contaminated after reprocessing include the device's design; breaches of recommended reprocessing guidelines (eg, inadequate manual cleaning, delayed reprocessing and/or improper device storage); damage to the device; and/or lacking servicing, maintenance or repair (table 1 and table 2 ).

In August 2015, the FDA issued a safety communication advising that it is "aware of instances of persistent bacterial contamination (of duodenoscopes) even following strict adherence to manufacturer reprocessing instructions." ${ }^{14}$ To reduce the infection risk, this communication recommended healthcare facilities performing ERCP consider at least one of four supplemental measures "in addition to meticulous cleaning as part of strict adherence to the (duodenoscope) manufacturer's reprocessing instructions": EO gas sterilisation, microbiological culturing, use of a liquid chemical sterilant processing system and repeating high-level disinfection. ${ }^{14}$ This FDA communication further advised that "[w] hen possible and practical, duodenoscopes should be sterilised due to the greater margin of safety provided by sterilisation. Sterilisation is a validated process used to render a product free from all viable microorganisms." Humphries et al similarly reported that in their opinion, "the complexity of modern duodenoscopes necessitates the classification of these devices as 'critical' and should require sterilization. ${ }^{20}$ Whether the FDA and CDC might reclassify duodenoscopes as critical devices requiring sterilisation is currently unresolved. ${ }^{6}{ }^{15}$ (In this August 2015 communication, the FDA acknowledged that these four supplemental measures "may not be feasible in all health care facilities and each of these options comes with its own benefits and limitations." ${ }^{14}$ )

Moreover, while some risk assessments have concluded that the transmission of infection by a GI endoscope is $\operatorname{rare}^{234}$ (eg, 1 case per 1.8 million procedures $\left.{ }^{34}\right)$, the research of others suggests that the true incidence of infections linked to GI endoscopes could be significantly higher. ${ }^{152035}$ Whether some transmissions in past years may have been "masked" by antibiotics, but are now being more readily detected due, in part, to an organism's evolved resistance to carbapenems and other lastresort antibiotics, is unclear but possible. Also a possible contributor to underreporting the infection rate might be true cases of cross-infection misattributed to the translocation of the patient's own endogenous bacterial flora. Gastmeier and Vonberg reported that it is likely that infections and outbreaks (at least those caused by Klebsiella spp. bacteria) "have been missed in the past because this pathogen belongs to the physiological gut flora." ${ }^{36}$

Humphries $e t$ al discussed the possibility of true infections being overlooked and not recorded due to insufficient detection mechanisms, writing that there are "concerns that duodenoscope-related infections may be underestimated." ${ }^{20}$ These authors further noted that "[m] ost hospitals do not perform postprocedure surveillance for infections and would not be able to identify an outbreak from baseline postprocedural infection rates," adding that reports of outbreaks due to CRE "probably predominate largely because these organisms are flagged for additional investigation by hospital laboratories. However, clusters due to susceptible bacteria may be missed." ${ }^{20}$ Wang et al investigated the rates of infections after colonoscopy and esophagogastroduodenoscopies performed in US ambulatory surgery centres, reporting similarly that "postendoscopic infections are more common than previously thought and vary widely by facility" and that "postendoscopic infections are occurring without being detected by existing surveillance systems." ${ }^{\prime 5}$ Other factors that can cause published 


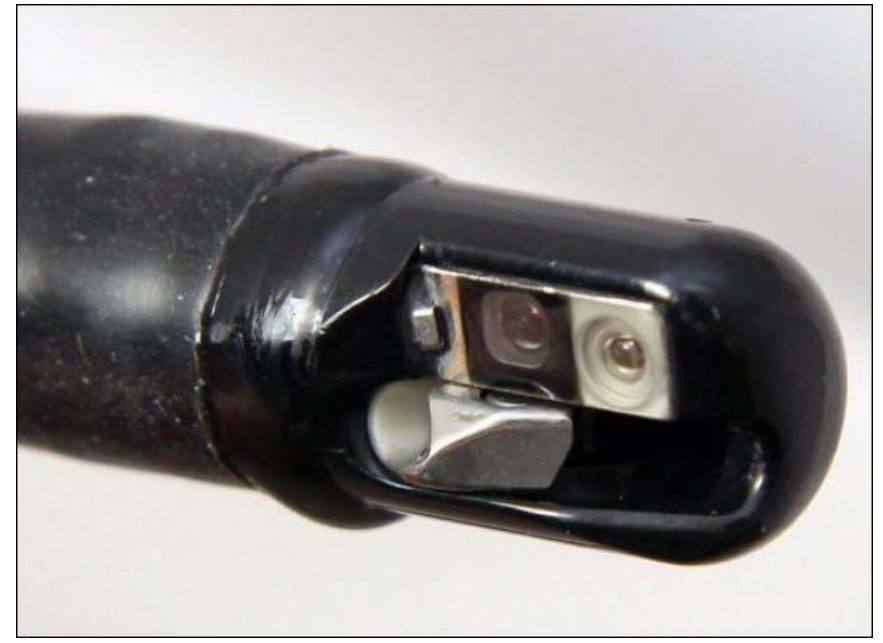

Figure 1 Pictured is the distal end of a duodenoscope featuring a sealed elevator-wire channel. Behind this mechanism is a recessed area. This mechanism connects to a knob on the duodenoscope's control head via a wire (neither visible) that is housed in the sealed elevator-wire channel (also not visible) (Source: FDA).

estimates of the infection rate to understate the true risk include these estimates' general failure to include in their calculations infections filed with the FDA in the form of a regulatory report (as opposed to being published in a peer-reviewed journal) and undisclosed infections.

The duodenoscope's design: Displayed in figure 1, duodenoscopes feature a forceps elevator mechanism at their distal end that is used to help thread a catheter, guidewire or other accessory into the common biliary duct during ERCP. ${ }^{8}$ A long, enclosed wire connects this mechanism to a manually controlled knob on the duodenoscope's control section that is used to adjust this mechanism and alter the direction of the accessory during the procedure. In some older duodenoscope models, this wire is housed in a narrow channel that is open and requires reprocessing. ${ }^{8}$ In newer models, however, this elevator-wire channel is designed to be sealed at the duodenoscope's distal end obviating the channel's reprocessing. ${ }^{7811}$

While crucial to the success of ERCP, however, the FDA and others have reported that the design of the duodenoscope's forceps elevator mechanism and distal end can hinder cleaning. ${ }^{5} 101314$ The FDA's February 2015 safety communication acknowledged "effective cleaning of all areas of the duodenoscope may not be possible." ${ }^{13}$ This communication further stated that moving parts of this elevator mechanism may remain contaminated after cleaning and disinfection. Others have similarly discussed the potential for the duodenoscope's distal end to remain contaminated despite being reprocessed according to duodenoscope reprocessing guidelines. ${ }^{7-14} 37$ In addition to other discussed factors, biofilms forming inside the duodenoscope and resisting removal with a cleaning brush can adversely impact the effectiveness of reprocessing. $^{711182225}$

Comparative effectiveness of the FDA's four supplemental measures: Rubin et al discussed some notable advantages and disadvantages of each of the four supplemental measures the FDA suggested in August 2015 that hospitals performing ERCP consider implementing to improve the effectiveness of duodenoscope reprocessing. ${ }^{22}$ Published comparisons of the effectiveness, feasibility and cost of each of these four measures, however, are generally scant. In addition to EO gas being a common option available for sterilising heat-sensitive endoscopes, ${ }^{1}$ Rubin et al wrote that the "clinical success of (EO gas) sterilisation has been shown in several instances in which implementation led to immediate cessation of bacterial outbreaks," 22 which is consistent with this review's findings. Some unrelated outbreak investigations have reported similar findings vis-a-vis the EO gas sterilization of bronchoscopes. ${ }^{2138}$

Using one of the FDA's other supplemental measures, a hospital in Washington state reported microbiologically culturing (and quarantining) duodenoscopes to improve safety. ${ }^{12}$ Other measures and mitigations that this review identified for preventing additional transmissions of multidrug-resistant bacteria include (of course) removing the implicated endoscope from clinical use and returning the endoscope to the manufacturer for servicing and, as required, repair. ${ }^{12192039}$ Use of a borescope to inspect the working channel of the endoscope for visible contamination, biofilms and/or defects prior to the endoscopic procedure could potentially also improve safety. ${ }^{1922}$ Another option for evaluating an endoscope for contamination with organic soil, as well as for training and cleaning competency testing, are test kits designed to detect haemoglobin, protein, carbohydrates or adenosine triphosphate, also known as ATP. ${ }^{62234}$

More about EO gas sterilisation: According to the CDC, EO gas "has been the most commonly used process for sterilising temperature- and moisture-sensitive medical devices and supplies in healthcare institutions in the United States." ${ }^{\prime}$ Several low-temperature processes including those using EO gas have been cleared by the FDA for sterilising flexible endoscopes, including bronchoscopes and choledocoscopes. Some of these devices may be labelled soon for the sterilisation of duodenoscopes too, as manufacturers endeavor to develop effective technologies to increase the safety of ERCP. Like all low-temperature sterilisation technologies, however, EO gas sterilisation has limitations. For example, it may not be available in the healthcare facility, ${ }^{1622}$ and while an EO gas steriliser's exposure cycle may be only a few hours in duration, the processed instruments may require an aeration time of 12 hours or more prior to reuse. Consequently, medical facilities using this sterilisation method may have to purchase additional duodenoscopes to maintain an adequate inventory and meet patient demand. Assessing the impact of this expense in the context of the financial costs associated with outbreaks of CRE and MDROs, however, has been suggested. ${ }^{17}$

Notwithstanding the FDA's advice that this method be considered (along with three other enhanced measures) to improve the safety of duodenoscopes, ${ }^{14}$ claims that 
EO gas sterilisation may damage a flexible endoscope over time have been reported. ${ }^{40}$ However, other studies have found EO gas not to damage the duodenoscope or to have deleterious effects on the materials used in its construction. ${ }^{141}$ Moreover, while the FDA may not have cleared a device, per se, that uses EO gas (or most other low-temperature chemicals) specifically to sterilise duodenoscopes, some endoscope manufacturers (as described in the device's operating and reprocessing instructions) identify EO gas as a technology that may be used to achieve sterilization. Indeed, this review found EO gas to have emerged as an effective option for sterilizing duodenoscopes and preventing additional infections (table 1).

Published concerns about certain safety risks associated with EO gas warrant discussion. ${ }^{12242}$ It may be useful, however, to assess the potential significance of these risks in the context of safety concerns associated with other reprocessing methods. To be sure, not only EO gas, but most other chemical agents used to disinfect or sterilise a reusable device can present a risk to healthcare staffers, patients and/or the environment, if not properly mitigated. ${ }^{43-47}$ Even detergents, and tap water too, used to clean and rinse reusable devices, respectively, can pose a safety risk (again, unless these risks are adequately mitigated). ${ }^{48}{ }^{49}$ Education, training and compliance with the manufacturer's instructions, among other measures, are important to reduce, if all but eliminate, the potential risks associated with any disinfection or sterilisation process or reprocessing technology, not only EO gas.

\section{RECOMMENDATIONS}

Several recommendations are provided in box 1 , based on this review's specific findings, to improve the safety of ERCP and reduce the risk of a duodenoscope transmitting CRE or a related MDRO. Several of these recommendations may be applied to other types of flexible endoscopes, too. The importance of successfully reprocessing all types of flexible endoscopes cannot be overstated, as more healthcare-associated outbreaks have been linked to contaminated flexible endoscopes than to any other type of reusable medical instrument. ${ }^{1}$

\section{CONCLUSIONS}

The review's findings suggest that, due to a number of factors, current reprocessing practices may not always be sufficiently effective to prevent a duodenoscope from

Box 1 Recommendations to reduce the risk of a duodenoscope transmitting carbapenem-resistant Enterobacteriaceae (CRE) or a related multidrug-resistant organism (MDRO) during endoscopic retrograde cholangiopancreatography (ERCP). Several of these recommendations may be applied to and considered for other types of flexible endoscopes, too:

- Meticulously clean the duodenoscope prior to high-level disinfection or sterilisation (in accordance with the device manufacturer's reprocessing instructions).

- Pre-clean and then thoroughly reprocess the device promptly after the endoscopic procedure. (If warranted, follow the manufacturer's "delayed reprocessing" instructions. ${ }^{52}$ )

- Consider using a borescope to inspect the working channel of device for visible contamination, moisture, biofilms and/or damage. ${ }^{1922}$

- Test kits designed to detect haemoglobin, protein, carbohydrates, total organic carbon or adenosine triphosphate (ATP) may also be used to assess and audit the cleaning process. ${ }^{62235}$

- When feasible and practical, consider sterilising the duodenoscope (using a process validated for effectiveness and materials' compatibility). ${ }^{514}$

- Clinical data collected in outbreak settings indicate that EO gas can be an effective tool and option for sterilizing duodenoscopes and terminating outbreaks of CRE and related MDROs (table 1).

- Despite the greater margin of safety availed by sterilisation (compared to disinfection), cleaning of the duodenoscope first remains essential.

- The following factors (among others) can adversely impact the effectiveness of reprocessing:

- Improperly cleaning the device, including failure to clean the device promptly after the endoscopic examination. ${ }^{53}$

- A device's complex design that may hinder cleaning. ${ }^{911} 1314$

- Damage to the device, or failure to maintain, service and/or repair the device according to the manufacturer's instructions. ${ }^{1220}$

- Formation of a resistive biofilm inside the device, resulting in the device's persistent contamination with bacteria (eg, CRE or a related MDROs) sometimes despite cleaning. ${ }^{67911142238}$

- Inadequate drying, or storing the device in a unclean, moist or poorly ventilated area.

- If sterilisation is not performed, then meticulously clean, high-level disinfect and dry (and store) the duodenoscope in strict accordance with the manufacturer's reprocessing instructions (which may be supplemented with published guidelines for reprocessing gastrointestinal endoscopes). ${ }^{1-3}$

- Liquid chemical sterilants/disinfectants, such as glutaraldehyde, peracetic acid and ortho-phthalaldehyde, are commonly used to high-level disinfect flexible endoscopes, usually in less than an hour, and sometimes within a few minutes. ${ }^{1}$

- Consider adopting at least one of the FDA's other suggested supplemental measures (eg. microbiological culturing) to enhance the effectiveness of duodenoscope reprocessing. ${ }^{14}$

- Remember that the FDA's four supplemental measures are to be combined with and to follow (not replace) a healthcare facility's strict adherence to the endoscope manufacturer's reprocessing instructions (ie, meticulous manual cleaning and high-level disinfection). ${ }^{14}$

- The FDA recognises that "not all healthcare facilities can implement one or more of these measures, which require specific resources, training, and expertise. Therefore, it is critical that staff responsible for reprocessing duodenoscopes have the manufacturer's instructions readily available to promote strict adherence to the reprocessing instructions in the device labeling, understand the importance of their role in reprocessing the device, and maintain proficiency in performing these reprocessing tasks." ${ }^{14}$ 
transmitting CRE or a related MDRO, at least in some circumstances including an outbreak setting (tables 1 and 2). Factors that may contribute to the device remaining contaminated after reprocessing include the device's design; breaches of recommended reprocessing guidelines (eg, inadequate manual cleaning, delayed reprocessing or improper device storage); damage to the device; lacking servicing, maintenance or repair; and/or the presence of resistive biofilms. More research is recommended to better identify and characterize the potential contribution of each of these (and other factors) to the transmission of CRE and MDROs during ERCP. (Note: High-level disinfection can be expected to prevent transmission of CRE and their related MDROs provided the high-level disinfectant/sterilant contacts all of the device's contaminated surfaces. $)^{8}$ Measures this review identified that can mitigate the impact of these and other reprocessing challenges and reduce, if not eliminate, the risk of a duodenoscope transmitting these multidrugresistant bacteria include the use of EO gas sterilization, among other effective measures. The FDA has suggested that healthcare facilities consider performing at least one of four supplemental measures, which include EO gas sterilisation, to improve the effectiveness of duodenoscope reprocessing. Other measures adopted to reduce the infection risk include removing the implicated device from use, re-training of staff about proper cleaning, microbiological culturing and returning the device to the manufacturer for evaluation, servicing and/or repair. More research is also recommended to develop additional measures and options that prevent infections of CRE and related MDROs following ERCP. Whether the FDA and CDC might reclassify duodenoscopes as critical devices requiring sterilisation is currently unresolved.

Acknowledgements Figure 1 was obtained from the FDA's website. Stated on the FDA's website (https://www.fda.gov/aboutfda/aboutthiswebsite/websitepolicies/ default.htm), "[u]nless otherwise noted, the contents of the FDA website (www. fda.gov) - both text and graphics—are not copyrighted. They are in the public domain and may be republished, reprinted and otherwise used freely by anyone without the need to obtain permission from FDA. Credit to the US Food and Drug Administration as the source is appreciated but not required."

Contributors LFM of LFM Healthcare Solutions, LLC is the sole researcher, planner, organiser and author of this article and all of its content including tables. LFM is the president of LFM Healthcare Solutions LLC, which is an independent safety and quality improvement company.

Funding This article was sponsored by Andersen Products, Inc. (Haw River, NC), who owns this article's copyright.

Competing interests This article discusses low-temperature sterilisation technologies in general and ethylene oxide gas sterilisation in particular. Andersen Products manufactures ethylene oxide gas sterilisers. While LFM's company has received research sponsorship in the past from companies that market sterilisation technologies including Andersen Products, disposable devices and reusable endoscopes, none contributed to or participated in, directly or indirectly, LFM's writing of this article.

Patient consent for publication Not required.

Provenance and peer review Not commissioned; externally peer reviewed.

Data availability statement All data relevant to the study are included in the article or uploaded as supplementary information.

Open access This is an open access article distributed in accordance with the Creative Commons Attribution Non Commercial (CC BY-NC 4.0) license, which permits others to distribute, remix, adapt, build upon this work non-commercially, and license their derivative works on different terms, provided the original work is properly cited, appropriate credit is given, any changes made indicated, and the use is non-commercial. See: http://creativecommons.org/licenses/by-nc/4.0/.

ORCID iD

Lawrence F Muscarella http://orcid.org/0000-0001-9795-6313

\section{REFERENCES}

1 Centers for Disease Control and Prevention (CDC). Guideline for disinfection and sterilization in healthcare facilities, 2008.

2 Calderwood AH, Day LW, Muthusamy VR, et al. ASGE guideline for infection control during $\mathrm{Gl}$ endoscopy. Gastrointest Endosc 2018;87:1167-79.

3 Society of Gastroenterology Nurses and Associates (SGNA). Guideline for use of high-level disinfectants and sterilants for reprocessing flexible gastrointestinal endoscopes, 2013.

4 Spaulding. EH The role of chemical disinfection in the prevention of nosocomial infections. In: Brachman PS, Eickoff TC, eds. Proceedings of the International Conference on nosocomial infections, 1970. Chicago: American Hospital Association, 1971: 254-74.

5 Food and Drug Administration (FDA). Reprocessing medical devices in health care settings: validation methods and labeling guidance for industry and food and drug administration staff, 2015: 1-40.

6 Petersen BT, Cohen J, Hambrick RD, et al. Multisociety guideline on reprocessing flexible Gl endoscopes: 2016 update. ASGE reprocessing guideline Task Force. Gastrointest Endosc 2017;85:282-94.

7 United States Senate Health, Education, Labor and Pensions (HELP) Committee. Patty Murray, Ranking Member. Preventable Tragedies: Superbugs and How Ineffective Monitoring of Medical Device Safety Fails Patients., 2016.

8 Muscarella LF. Risk of transmission of carbapenem-resistant Enterobacteriaceae and related "superbugs" during gastrointestinal endoscopy. World J Gastrointest Endosc 2014;6:457-74.

9 Epstein L, Hunter JC, Arwady MA, et al. New Delhi metallo$\beta$-lactamase-producing carbapenem-resistant Escherichia coli associated with exposure to duodenoscopes. JAMA 2014;312:1447-55.

10 Centers for Disease Control and Prevention (CDC). Notes from the Field: New Delhi metallo- $\beta$-lactamase-producing Escherichia coli associated with endoscopic retrograde cholangiopancreatography Illinois, 2013. MMWR Morb Mortal Wkly Rep 2014;62:1051.

11 Verfaillie CJ, Bruno MJ, Voor in 't Holt AF, et al. Withdrawal of a novel-design duodenoscope ends outbreak of a VIM-2-producing Pseudomonas aeruginosa. Endoscopy 2015;47:493-502.

12 Wendorf KA, Kay M, Baliga C, et al. Endoscopic retrograde cholangiopancreatography-associated AmpC Escherichia coli outbreak. Infect Control Hosp Epidemiol 2015;36:634-42.

13 Food and Drug Administration (FDA). Design of endoscopic retrograde cholangiopancreatography (ERCP) Duodenoscopes may impede effective cleaning: FDA Safety communication. FDA, 2015.

14 Food and Drug Administration (FDA). Supplemental measures to enhance duodenoscope reprocessing: FDA Safety communication. FDA, 2015

15 Association for the Advancement of Medical Instrumentation (AAMI). Strong evidence for sterilization of endoscopes presented at Stakeholder meeting, 2017.

16 Smith ZL, Oh YS, Saeian K, et al. Transmission of carbapenemresistant Enterobacteriaceae during ERCP: time to revisit the current reprocessing guidelines. Gastrointest Endosc 2015;81:1041-5.

17 Naryzhny I, Silas D, Chi K. Impact of ethylene oxide gas sterilization of duodenoscopes after a carbapenem-resistant Enterobacteriaceae outbreak. Gastrointest Endosc 2016;84:259-62.

18 Rauwers AW, Voor In 't Holt AF, Buijs JG, et al. High prevalence rate of digestive tract bacteria in duodenoscopes: a nationwide study. Gut 2018;67:1637-45.

19 Parr A, Querry A, Pasculle A, et al. Carbapenem-resistant Klebsiella pneumoniae cluster associated with gastroscope exposure among surgical intensive care unit patients at University of Pittsburgh Medical Center. Open Forum Infect Dis 2016;3(suppl_1). Fall.

20 Humphries RM, Yang S, Kim S, et al. Duodenoscope-related outbreak of a carbapenem-resistant Klebsiella pneumoniae identified using advanced molecular diagnostics. Clin Infect Dis 2017;65:1159-66.

21 Mehta A, Muscarella LF. Updated guidance to prevent bronchoscope-associated infections of "superbugs": A comprehensive review. Submitted for publication. 
22 Rubin ZA, Kim S, Thaker AM, et al. Safely reprocessing duodenoscopes: current evidence and future directions. Lancet Gastroenterol Hepatol 2018;3:499-508.

23 Marsh JW, Krauland MG, Nelson JS, et al. Genomic epidemiology of an endoscope-associated outbreak of Klebsiella pneumoniae carbapenemase (KPC)-producing K. pneumoniae. Plos One 2015;10:e0144310.

24 McCool S, Querry A, Muto C, et al. High level disinfection failure in gastrointestinal scopes with elevator channels - is it time to switch to ethylene oxide (ETO) sterilization? Open Forum Infect Dis 2014;1(suppl_1).

25 Food and Drug Administration (FDA). Brief summary of the gastroenterology and urology devices panel meeting, 2015.

26 Shenoy ES, Pierce VM, Walters MS, et al. Transmission of mobile colistin resistance (mcr-1) by duodenoscope. Clin Infect Dis 2018.

27 Pasadena Public Health Department. Investigation of an outbreak of multi-drug resistant Pseudomonas aeruginosa following ERCP at Huntington Hospital, 2016: 1-11.

28 Food and Drug Administration (FDA). MAUDE database. Regulatory report number: 2951238-2015-00347, 2015.

29 Alrabaa SF, Nguyen P, Sanderson R, et al. Early identification and control of carbapenemase-producing Klebsiella pneumoniae, originating from contaminated endoscopic equipment. Am J Infect Control 2013;41:562-4.

30 Muscarella LF. Investigation and prevention of infectious outbreaks during endoscopic retrograde cholangiopancreatography. Endoscopy 2010;42:957-9.

31 Terhune C. Superbug linked to 2 deaths at UCLA Hospital; 179 potentially exposed. Los Angeles Times, 2015

32 Centers for Disease Control and Prevention (CDC). Interim protocol for healthcare facilities regarding surveillance for bacterial contamination of duodenoscopes after reprocessing 2015.

33 Food and Drug Administration (FDA). MAUDE database. Regulatory report number: 9610877-2017-00417, 2017.

34 Komanduri S, Abu Dayyeh BK, Bhat YM, et al. Technologies for monitoring the quality of endoscope reprocessing. ASGE technology Committee. Gastrointest Endosc 2014;80:369-73.

35 Wang P, Xu T, Ngamruengphong S, et al. Rates of infection after colonoscopy and osophagogastroduodenoscopy in ambulatory surgery centres in the USA. Gut 2018;67:1626-36.

36 Gastmeier P, Vonberg R-P. Klebsiella spp. in endoscopy-associated infections: we may only be seeing the tip of the iceberg. Infection 2014;42:15-21.

37 Food and Drug Administration (FDA). Infections associated with reprocessed duodenoscopes. (A webpage). Updated 2017.

38 Alipour N, Karagoz A, Taner A, et al. Outbreak of hospital infection from biofilm-embedded pan drug-resistant Pseudomonas aeruginosa, due to a contaminated bronchoscope. J Prev Med 2017;02:1-9.

39 Zweigner J, Gastmeier P, Kola A, et al. A carbapenem-resistant Klebsiella pneumoniae outbreak following bronchoscopy. Am J Infect Control 2014;42:936-7.

40 Willman D, Terhune C. FDA official casts doubt on new method to clean scopes linked to infections. Los Angeles Times, 2015.

41 Smith ZL, Dua A, Saeian K, et al. A novel protocol obviates endoscope sampling for carbapenem-resistant Enterobacteriaceae: experience of a center with a prior outbreak. Dig Dis Sci 2017;62:3100-9.

42 Boiano JM, Steege AL. Ethylene oxide and hydrogen peroxide gas plasma sterilization: precautionary practices in U.S. hospitals. Zentralsterilisation 2015;23:262-8.

43 Ballantyne B, Jordan SL, Toxicological JSL. Toxicological, medical and industrial hygiene aspects of glutaraldehyde with particular reference to its biocidal use in cold sterilization procedures. $J$ Appl Toxicol 2001;21:131-51.

44 Shih H-Y, Wu D-C, Huang W-T, et al. Glutaraldehyde-induced colitis: case reports and literature review. Kaohsiung J Med Sci 2011;27:577-80.

45 Cooper DE, White AA, Werkema AN, et al. Anaphylaxis following cystoscopy with equipment sterilized with Cidex OPA (ortho-phthalaldehyde): a review of two cases.. J Endourol 2008;22:2181-4.

46 Centers for Disease Control and Prevention (CDC). Health risks to workers associated with occupational exposures to peracetic acid; Request for information. Federal Register;82:12819-21.

47 Centers for Disease Control and Prevention (CDC). Corneal decompensation after intraocular ophthalmic surgery--Missouri, 1998. MMWR Morb Mortal Wkly Rep 1998;47:306-8.

48 Muscarella LF. Enzymatic detergents: are they the primary cause of TASS? Insight 2018;43.

49 Muscarella LF. Inconsistencies in endoscope-reprocessing and infection-control guidelines: the importance of endoscope drying Am J Gastroenterol 2006;101:2147-54.

50 Johnson SR. CRE superbug hits in NC; endoscopes not pinpointed as culprit this time. Modern Healthcare 2015.

51 Olinger DE. Denver Post E. coli outbreak that infected 9 at UCH occurred over seven weeks, 2016.

52 Food and Drug Administration (FDA). MAUDE database. Regulatory report number: 2951238-2019-00470, 2019.

53 Kola A, Piening B, Pape U-F, et al. An outbreak of carbapenemresistant OXA-48--producing Klebsiella pneumonia associated to duodenoscopy. Antimicrob Resist Infect Control 2015;4.

54 Food and Drug Administration (FDA). MAUDE database. Regulatory report number: 8010047-2018-00601, 2018. 


\section{Correction: Use of ethylene-oxide gas sterilisation to terminate multidrug-resistant bacterial outbreaks linked to duodenoscopes}

Muscarella LF. Use of ethylene-oxide gas sterilisation to terminate multidrugresistant bacterial outbreaks linked to duodenoscopes. BMJ Open Gastroenterol 2019;6:e000282. doi: 10.1136/bmjgast-2019-000282.

The article has been corrected since it is published.

1. In Abstract's 'Conclusions', 'Factors this review identifed' has been corrected to 'Factors this review identified'.

2. In Table 1, in the column 'Case's implemented measure(s), response', 'Began culturing, quaranting duodenoscopes' has been corrected to 'Began culturing, quarantining duodenoscopes'.

3. In the section 'More about EO gas sterilisation' under 'Discussion', the spelling of 'endeavour' has been changed to 'endeavor' in the sentence 'Some of these devices may be labelled soon for the sterilisation of duodenoscopes too, as manufacturers endeavour to develop effective technologies to increase the safety of ERCP.'

4. 'ie,' and 'eg,' have been used consistently throughout the article.

Open access This is an open access article distributed in accordance with the Creative Commons Attribution Non Commercial (CC BY-NC 4.0) license, which permits others to distribute, remix, adapt, build upon this work non-commercially, and license their derivative works on different terms, provided the original work is properly cited, appropriate credit is given, any changes made indicated, and the use is non-commercial. See: http:// creativecommons.org/licenses/by-nc/4.0/.

(c) Author(s) (or their employer(s)) 2019. Re-use permitted under CC BY-NC. No commercial re-use. See rights and permissions. Published by BMJ.

BMJ Open Gastro 2019;6:e000282corr1. doi:10.1136/bmjgast-2019-000282corr1

A) Check for updates 